\title{
InP HEMT外延结构材料抗电子辐照加固设计研究*
}

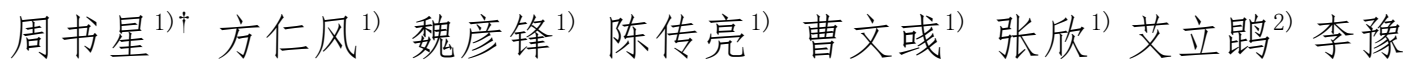
东 $^{3)}$ 郭旗 ${ }^{3)}$

1)（湖北文理学院物理与电子工程学院, 低维光电材料与器件湖北省重点实验室, 襄阳 441000)

2) (中国科学院上海微系统与信息技术研究所, 信息功能材料国家重点实验室, 上海 200050)

3) (中国科学院新疆理化技术研究所, 中国科学院特殊环境功能材料与器件重点实验室, 乌 鲁木齐 830011)

\section{摘 要}

为研究磷化铟高电子迁移率晶体管(InP HEMT)外延结构材料的抗电子辐照 加固设计, 本文采用气态源分子束外延法制备了系列InP HEMT外延结构材料, 针对不同外延结构材料开展了 $1.5 \mathrm{MeV}$ 电子束辐照试验, 辐照注量为 $2 \times 10^{15} \mathrm{~cm}^{-2}$, 并测试了InPHEMT外延结构材料二维电矛 ( 2 (2EG) 辐照前后的电学特性, 获 得了辐照前后不同外延结构InP HEMT材料维电子气归一化浓度和电子迁移 率随外延参数的变化规律, 分析了 InPHEMT二维电子气辐射损伤与外延结构参 数 (如 $\mathrm{Si}-\delta$ 掺杂浓度、 $\mathrm{InGaAs}$ 沟道厚度和沟道In组分以及隔离层厚度等结构参 数) 依赖的关系。结果表明; Si- $\delta$ 掺杂浓度越大, 隔离层厚度较薄, InGaAs 沟道 厚度较大, 沟道In组分低的InP HEMT外延结构二维电子气辐射损伤相对较低, 具有更强的抗电子辎照能力。经分析原因如下：1）电子束与材料晶格发生能量 传递, 破坏晶格完整性, 且在沟道异质界面引入辐射诱导缺陷, 增加复合中心密 度, 散射增强导致一维电子气迁移率和浓度降低. 2) 高浓度 $\mathrm{Si}-\delta$ 掺杂和薄隔离层 有利于提高量子阱二维电子气浓度，降低二维电子气受辐射损伤的影响. 3) 高 In组分应变沟道有利于提高二维电子气迁移率，但辐照后更容易应变弛豫产生 位错缺陷, 导致二维电子气迁移率显著下降。

关键词: InP HEMT; 二维电子气; 电子束辐照; 辐射加固

PACS: 72.80.Ey, 71.10.Ca, 61.82.-d, 61.80.-x

*国家自然科学基金(批准号: 11705277, 61434006)和湖北文理学院博士科研启动基金 (批准 号: kyqdf2059038)资助的课题. 
†通信作者. E-mail: sxzhou2020@163.com

\section{1 引言}

随着载人航天嫦娥探月工程和火星探测任务的开启, 航天探测工程步入到深 空阶段, 深空探测系统需要传输的探测数据越来越多, 通信信号越来越弱, 通信 时延增加, 急需信噪比高, 灵敏度高、工作频率高的深空通信收发系统。磷化铟 基高电子迁移率晶体管（InP based High Electron Mobility Transistor, InP HEMT) 利用 $\mathrm{InGaAs}$ 沟道具有的高迁移率的二维电子气(2DEG)来工作, 使其具有高速高 频、高功率增益、低噪声及低功耗等特点, 成为毫米波和太赫兹波领域最具竞争 力的三端固态电子器件之一, 非常适合制作毫米波太赫兹波低噪声放大器, 应用 于深空通信收发系统 ${ }^{[1-3]}$.

但是, 随着 HEMT 射频器件在空间环境中应用, 面临着包括地球辐射带、 太阳耀斑和银河宇宙射线等强辐射环境, 将不可辟免受到各种高能粒子和射线等 辐射影响, 其中电子是空间辐照环境中含量极为牛畐的粒子, 具有较大的电子能 损, 会在材料中引入微观缺陷, 并可在异质鱼面引起原子混合, 这都会造成 HEMT 量子阱中二维电子气迁移率和密度发生变化 ${ }^{[4-6]}$, 易于诱发空间通讯系统中 HEMT 射频器件的最小噪声系数 Fmin、截止频率 $\mathrm{ft}$ 、最大振荡频率 fmax 等关键 参数退化 ${ }^{[7-9]}$, 甚至器件失效, 影响空间通信的稳定性和准确性, 严重威胁航天器 的安全可靠运行. 因此对于空间环境中应用的 HEMT 器件, 研究其电子辐照效 应显得尤为重要.

近年来, 国外众多研究团队纷纷报道了辐照对各种 HEMT 器件的影响, 其 中辐照源包括质子、电子、中子、 $\gamma$ 射线、 $\alpha$ 粒子等, HEMT 器件主要包括 GaAs HEMT $^{[10]}$ GaNHEMT ${ }^{[11] 、 I n P ~ H E M T ~}{ }^{[12]}$ 、InAs/AlSb HEMT ${ }^{[13]}$. Pearton 等人发现 电子辐照会导致 GaHEMT 二维电子气面密度和电子迁移率减小, 阈值电压正向 漂移，同时漏电流和跨导退化 ${ }^{[14]}$. Fleetwood 等人发现沟道内缺陷及异质界面散 射会诱导载流子数量变化, 引起 HEMT 器件 $1 / \mathrm{f}$ 噪声增大 ${ }^{[8]}$. 林理涁等人分析了 电子质子辐照对 $\mathrm{AlGaAs} / \mathrm{GaAs} \mathrm{HEMT}$ 二维电子气密度和电子迁移率的辐射影响 规律和材料中引入的深能级缺陷, 发现外延结构和生长条件会影响 HEMT二维电 子气的抗辐照能力 ${ }^{[15,16]}$. 汤金金等人通过模拟仿真发现 GaN HEMT 异质结附近 $\mathrm{GaN}$ 层中的 $\mathrm{Ga}$ 空位会影响费米能级, 并且俘获势阱中二维电子气, 其中采用背 
部势垒层可以提高器件性能, 但会导致抗辐射性能降低 ${ }^{[5,17]}$. Z. Zang 等人质子辐 照实验发现: 辐照诱导阈值电压漂移与隔离层厚度正相关, 隔离层厚度较薄会提 高 GaN HEMT 抗辐射能力 ${ }^{[18]}$. M. D. Smith 等人实验发现: 通过材料外延设计和 钝化技术可以控制和提高 GaN HEMT 抗伽马辐照能力 ${ }^{[19]}$. 钟永辉等人通过模 拟仿真质子辐照 InP HEMT 辐照前后直流和射频特性变化发现：沟道下面插入一 层 $\mathrm{Si}-\delta$ 掺杂层可以增强质子辐射耐受性 ${ }^{[20]}$. 研究以上文献发现: 通过材料生长, 外延结构设计和工艺钝化技术都可以提高 HEMT 抗辐射性能, 但大部分主要集 中在 GaN HEMT 器件抗辐射效应研究上, 而 InP HEMT 器件抗辐射效应的研究 报道较少，在辐照损伤机理和抗辐射加固措施上有待进一步研究

二维电子气 (2DEG) 的电子迁移率 $(\mu)$ 和面密度 Ns 是影响 HEMT 器 件频率、功率、噪声系数等性能的两个重要参数, 研究辐射诱导缺陷对二维电子 气的影响对分析 HEMT 器件空间辐照退化机理和加固方法具有十分重要的意 义。目前国内在 InP HEMT 外延结构材料电子辐射效应方面的研究尚不够全面和 深入, 无法为 InP HEMT 器件与电路抗辐照加固设计提供理论指导. 因此本论文 拟对系列 InP HEMT 外延结构材料开展 1.5 MeV 电子束辐照试验, 获得辐照前后 二维电子气浓度和电子迁移率辐射损伤特性, 研究 InP HEMT 外延结构材料二维 电子气辐射与外延结构参数的相关性, 分析结构参数变化对 InP HEMT 外延结构 材料的抗辐照性能影响, 为 InP HEMT 器件与电路抗辐照加固设计提供数据支撑.

\section{2 材料制备与辐照试验}

\section{1 材料制备}

本实验所用 InP HEMT 外延结构材料样品是中国科学院上海微系统与信息 技术研究所提供的, 采用 V90 型气态源分子束外延生长系统(GSMBE)制备, 该 生长系统设备如图 1 所示. 样品基本结构如下表 1 所示, 外延结构从下至上的组 成为: $300 \mathrm{~nm}$ 非掺杂 $\operatorname{In}_{0.52} \mathrm{Al}_{0.48} \mathrm{As}$ 缓冲层(Buffer layer)、 $\mathrm{t}_{\mathrm{w}} \mathrm{nm}$ 非掺杂 $\mathrm{In}_{\mathrm{x}} \mathrm{Ga}_{1-\mathrm{x}} \mathrm{As}$ 沟道层(Channel)、 $\mathrm{h}_{\mathrm{w}} \mathrm{nm} \mathrm{In} 0.52 \mathrm{Al}_{0.48} \mathrm{As}$ 隔离层(Spacer layer)、Si- $\delta$ 平面掺杂层 $(\delta$ doping)、 $8 \mathrm{~nm}$ 非掺杂 $\mathrm{In}_{0.52} \mathrm{Al}_{0.48} \mathrm{As}$ 势垒层(Barrier layer)、 $4 \mathrm{~nm}$ 非掺杂 $\mathrm{InP}$ 停止刻 蚀层(Etch-stopper)、 $15 \mathrm{~nm}$ 的 $\mathrm{n}+$ 掺杂的 $\mathrm{In}_{0.52} \mathrm{Al}_{0.48} \mathrm{As}$ 帽层(Cap layer 3)、15nm 的 $\mathrm{n}+$ 掺杂的 $\mathrm{In}_{0.53} \mathrm{Ga} 0.47 \mathrm{As}$ 帽层(Cap layer 2) 和 $10 \mathrm{~nm}$ 的 $\mathrm{n}+$ 掺杂的 $\mathrm{In}_{0.65} \mathrm{Ga} 0.35 \mathrm{As}$ 帽层 (Cap layer 1). 为了研究 InP HEMT 二维电子气辐射损伤与外延结构参数依赖的 
关系, 进行抗辐射外延加固优化设计, 实验制备了四组不同结构参数的外延结构 样品. $\mathrm{A}$ 组样品的隔离层厚度为 $3 \mathrm{~nm}$, 沟道厚度为 $15 \mathrm{~nm}$, 沟道 $\mathrm{In}$ 组分为 0.53 , Si$\delta$ 平面掺杂层浓度分别为 $3 \times 10^{12} \mathrm{~cm}^{-2} 、 4 \times 10^{12} \mathrm{~cm}^{-2} 、 5 \times 10^{12} \mathrm{~cm}^{-2} 、 6 \times 10^{12} \mathrm{~cm}^{-2}$, 分 别标记为样品 A1、A2、A3、A4. B 组样品的 $\mathrm{Si}-\delta$ 平面掺杂层浓度为 $5 \times 10^{12} \mathrm{~cm}^{-}$ 2 , 沟道厚度为 $15 \mathrm{~nm}$, 沟道 $\mathrm{In}$ 组分为 0.53 , 隔离层厚度分别为 $2 \mathrm{~nm} 、 3 \mathrm{~nm} 、 4 \mathrm{~nm}$ 和 $5 \mathrm{~nm}$, 分别标记为样品 $\mathrm{B} 1 、 \mathrm{~B} 2 、 \mathrm{~B} 3 、 \mathrm{~B} 4 . \mathrm{C}$ 组样品的 $\mathrm{Si}-\delta$ 平面掺杂层浓度为 $5 \times 10^{12} \mathrm{~cm}^{-2}$, 隔离层厚度为 $3 \mathrm{~nm}$, 沟道厚度为 $10 \mathrm{~nm}$, 沟道 $\mathrm{In}$ 组分分别为 0.6 、 $0.65 、 0.7 、 0.75$, 分别标记为样品 $\mathrm{C} 1 、 \mathrm{C} 2 、 \mathrm{C} 3 、 \mathrm{C} 4 . \mathrm{D}$ 组样品的 $\mathrm{Si}-\mathrm{s}$ 平面掺杂 层浓度为 $5 \times 10^{12} \mathrm{~cm}^{-2}$, 隔离层厚度为 $3 \mathrm{~nm}$, 沟道 In 组分分别为 0.53 , 沟道厚度分 别为 $5 \mathrm{~nm} 、 15 \mathrm{~nm} 、 25 \mathrm{~nm}$ 和 $35 \mathrm{~nm}$, 分别标记为样品 D1、D2、D3、D4. 四组样品 辐照前常温(RT) 和低温 $77 \mathrm{~K}$ 霍尔测试结果如下表 2 所示. 图 2 表示沟道 In 组分 为 0.7 且沟道厚度为 $12 \mathrm{~nm}$ 的 InP HEMT 结构材料 TEM 分析图 ${ }^{[21]}$.

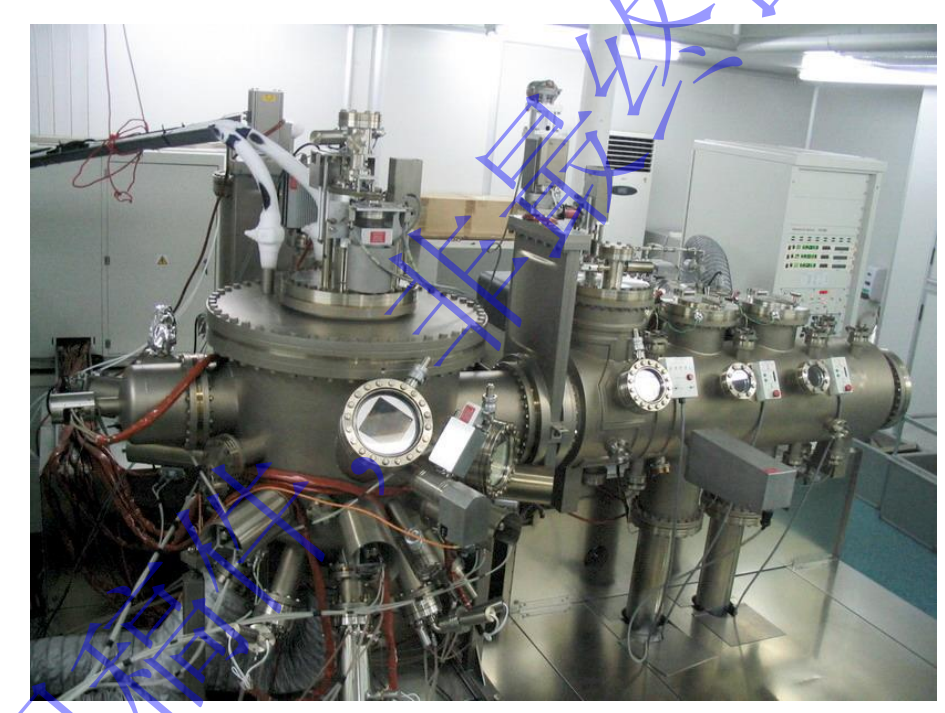

图1 V90型气态源分子束外延生长系统

Fig.1 V90 gas source molecular beam epitaxy growth system

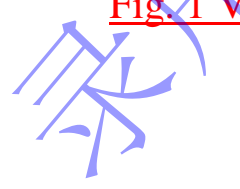

表 1 InP HEMT 外延结构表

Table.1 Structure parameters of the InP HEMT

\begin{tabular}{lccc}
\hline Layer & Material & Doping & Thickness(nm) \\
\hline Cap layer 1 & $\mathrm{In}_{0.65} \mathrm{Ga}_{0.35} \mathrm{As}$ & $\mathrm{N}+$ & 10 \\
Cap layer 2 & $\mathrm{In}_{0.53} \mathrm{Ga}_{0.47} \mathrm{As}$ & $\mathrm{N}+$ & 15 \\
Cap layer 3 & $\mathrm{In}_{0.52} \mathrm{Al}_{0.48} \mathrm{As}$ & $\mathrm{N}+$ & 15 \\
Etch-stopper & $\mathrm{InP}$ & $\mathrm{Un}$ & 4 \\
Barrier layer & $\mathrm{In}_{0.52} \mathrm{Al}_{0.48} \mathrm{As}$ & $\mathrm{Un}$ & 8 \\
-doping & $\mathrm{Si}$ & $3 \times 10^{12} \mathrm{~cm}^{-2} \sim 6 \times 10^{12} \mathrm{~cm}^{-2}$ \\
Spacer layer & $\mathrm{In}_{0.52} \mathrm{Al}_{0.48} \mathrm{As}$ & $\mathrm{Un}$ & $\mathrm{h}_{\mathrm{w}}$
\end{tabular}




\begin{tabular}{|c|c|c|c|}
\hline Channel & $\mathrm{In}_{\mathrm{x}} \mathrm{Ga}_{1-\mathrm{x}} \mathrm{As}$ & Un & $t_{w}$ \\
\hline Buffer layer & $\operatorname{In}_{0.52} \mathrm{Al}_{0.48} \mathrm{As}$ & Un & 300 \\
\hline \multicolumn{4}{|c|}{ S.I.InP sub } \\
\hline
\end{tabular}

表 2 InP HEMT 外延结构样品辐照前霍尔测试数据

Table. 2 Hall data of the InP HEMT structures before irradiation

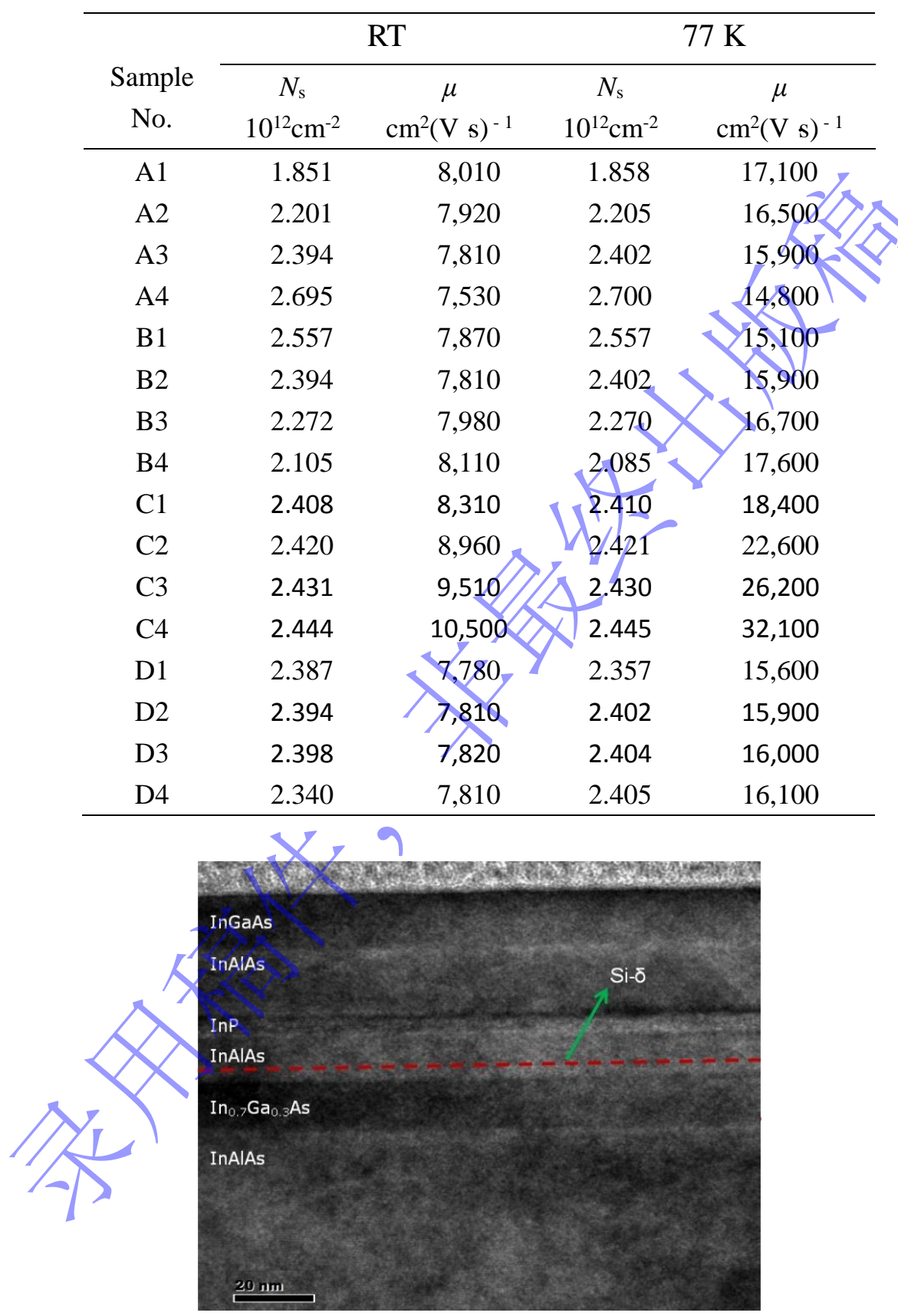

图2 InP HEMT结构材料TEM分析图

Fig. 2 TEM analysis diagram of InP HEMT structural material

\section{2 辐照试验}

电子辐照试验是在中国科学院新疆理化技术研究所完成的。试验采用的电子 
加速器型号为俄罗斯ELV-8II高频高压电子加速器, 能量范围为1.0 2.0 MeV。试 验选取入射电子能量为 $1.5 \mathrm{MeV}$, 注量率为 $1.0 \times 10^{11} \mathrm{~cm}^{-2} \cdot \mathrm{s}^{-1}$, 采用垂直入射方式. 当累积辐照注量达到 $2 \times 10^{15} \mathrm{~cm}^{-2}$ 时, 将样品取出进行常温和低温 $77 \mathrm{~K}$ 霍尔测试, 获取InP HEMT二维电子气浓度和迁移率.

\section{3 试验结果}

在辐照前后分别进行常温和低温77K霍尔测试, 获取InP HEMT二维电子气 浓度和迁移率, 并进行数据的归一化处理, 即采用辐照后的二维电子气浓度或电 子迁移率除以辐照前对应外延结构材料的二维电子气浓度或电子迁移率, 分析 InP HEMT外延结构材料二维电子气辐射损伤随外延结构参数的变化规律, 以便 进行外延结构加固设计优化.

图3表示电子辐照对不同 $\delta$ 掺杂浓度InP HEMT二维电子气浓度和电子迁移率 的影响. 从图3 (a) 和图3 (b) 中可以看到: 室温和 $77 \mathrm{~K}$ 二维电子气浓度和电子迁 移率辐射损伤趋势一致, 即在相同辐照条件下, 随着 $\delta$ 掺杂浓度增大, InP HEMT 外延结构二维电子气浓度和电子迁移率辐照损伤减小, 这说明 $\delta$ 掺杂浓度高的 InP HEMT外延结构二维电子气浓度和电乎移率辐照损伤相对较小, 具有较强的抗 辐照能力. 对比图3 (a) 和图3 (b) 可以发现: 同一样品在相同辐照条件下, $77 \mathrm{~K}$ 条件下的二维电子气浓度和电子迁移率比常温退化严重, 说明样品辐照后二维电 子气辐射损伤性能随着温度的降低会发生显著退化, 这主要是由于随着温度降低, 载流子热运动的平均速度变小, 不能较快的掠过辐射诱导缺陷, 偏转较大, 受到 缺陷散射增强, 且便客受到辐射诱导缺陷俘获 ${ }^{[22]}$. 图4表示电子辐照对不同隔 离层厚度InPHEMT二维电子气浓度和电子迁移率的影响. 从图4 (a) 和图4 (b) 中可以看到。室温和 $77 \mathrm{~K}$ 二维电子气浓度和电子迁移率辐射损伤趋势基本一致, 即在相同辐照条件下，随着隔离层厚度减小，InP HEMT外延结构二维电子气浓 度和电子迁移率辐照损伤快速减小, 这说明隔离层较薄的InP HEMT外延材料二 维电子气浓度和电子迁移率辐照损伤相对较小, 具有较强的抗辐照能力, 在 $\mathrm{GaN}$ HEMT中也观察到缩减隔离层厚度减小辐射退化的类似现象 ${ }^{[18]}$. 图 5 表示电子辐 照对不同沟道In组分InP HEMT二维电子气浓度和电子迁移率的影响. 从图5（a） 和图5（b）中可以看到：室温和 $77 \mathrm{~K}$ 二维电子气浓度和电子迁移率辐射损伤趋势 基本一致, 即在相同辐照条件下, 随着沟道In组分减小, InP HEMT外延结构二维 
电子气电子迁移率辐照损伤快速减小, 二维电子气浓度辐射损伤缓慢增大, 影响 较小, 这说明沟道In组分较小的InP HEMT外延材料二维电子气电子迁移率辐照 损伤相对较小, 具有较强的抗辐照能力. 图6表示电子辐照对不同沟道厚度 InP HEMT二维电子气浓度和电子迁移率的影响. 从图6 (a) 和图6（b) 中可以看到: 室温和 $77 \mathrm{~K}$ 二维电子气浓度和电子迁移率辐射损伤趋势基本一致, 即在相同辐照 条件下, 随着沟道厚度增大, InP HEMT外延结构二维电子气电子迁移率辐照损 伤缓慢减小, 二维电子气浓度辐射损伤变化不明显, 这说明沟道厚度大的 InP HEMT外延材料二维电子气电子迁移率辐照损伤相对较小, 具有较强的抗辐照能 力. 在图4、图5和图6中同样也可以发现：同一样品在相同辐照条件卡， $77 \mathrm{~K}$ 条 件下的二维电子气浓度和电子迁移率比常温退化严重, 这道啾无论样品的结构参 数如何, 其低温条件下的辐射损伤均较室温下严重。

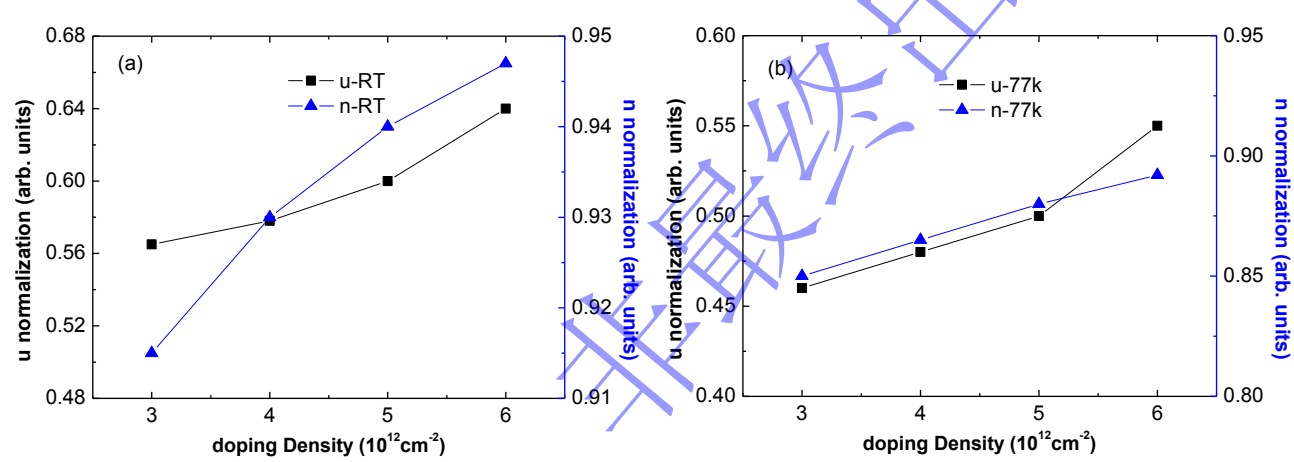

图3 电子辐照对不同 $\delta$ 掺杂浓度InP HEMT二维电子气浓度和电子迁移率的影响 (a) 室温; (b) $77 \mathrm{~K}$

Fig. 3 Normalized 2DEG density ( $\mathrm{N}_{\mathrm{S}}$ ) and electron mobility $(\mu)$ versus $\delta$-doping density measured at (a) room-temperature and (b) 77K irradiated by electron beam
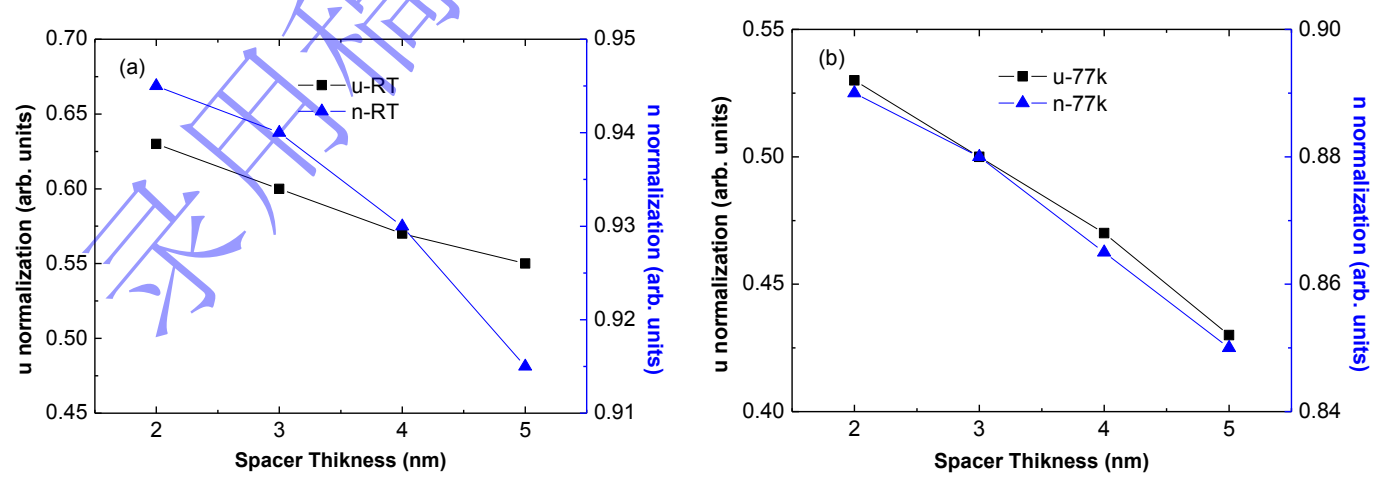

图4 电子辐照对不同隔离层厚度InP HEMT二维电子气浓度和电子迁移率的影响 (a) 室温; (b) $77 \mathrm{~K}$

Fig. 4 Normalized 2DEG density $\left(\mathrm{N}_{\mathrm{S}}\right)$ and electron mobility $(\mu)$ versus spacer layer thickness measured at (a) room-temperature and (b) $77 \mathrm{~K}$ irradiated by electron beam 

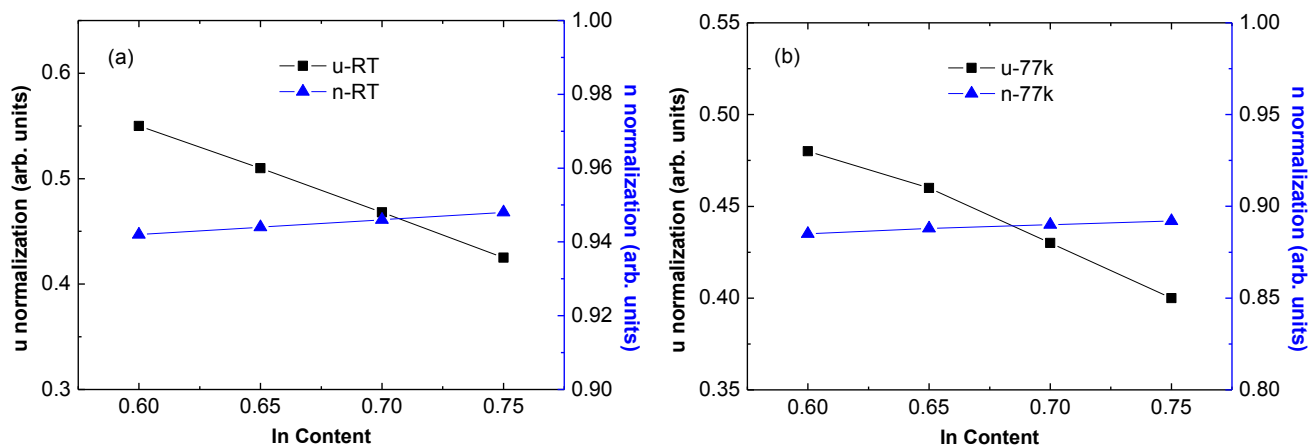

图5 电子辐照对不同InGaAs沟道In组分InP HEMT二维电子气浓度和电子迁移率 的影响（a）室温; (b) $77 \mathrm{~K}$

Fig. 5 Normalized 2DEG density $\left(\mathrm{N}_{S}\right)$ and electron mobility $(\mu)$ versus channel In content measured at (a) room-temperature and (b) $77 \mathrm{~K}$ irradiated by electron beam
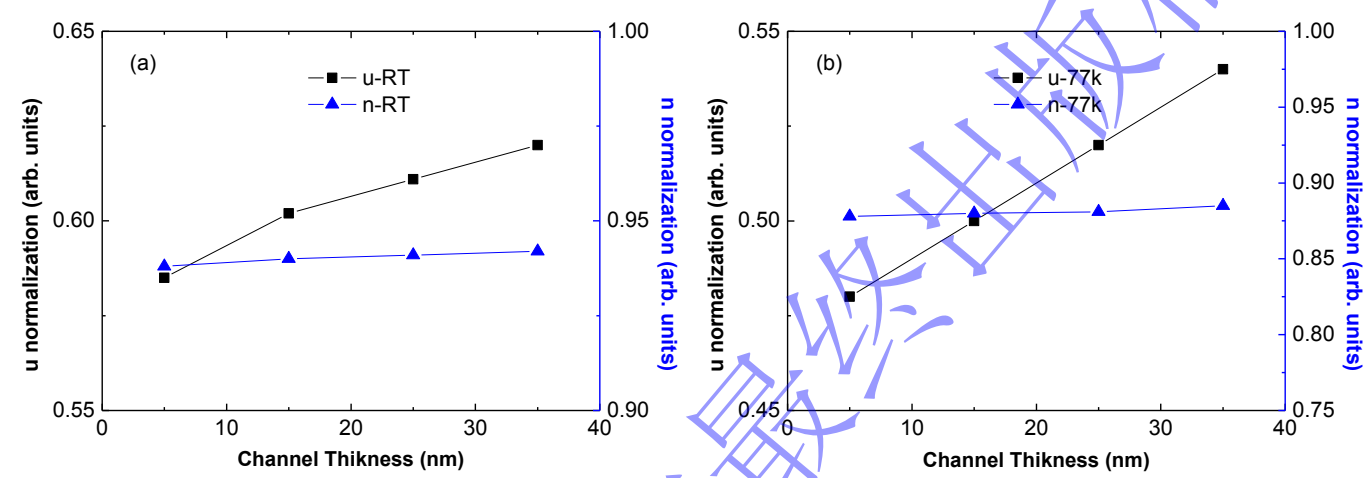

图6 电子辐照对不同沟道厚度 InP HEMT二维电子气浓度和电子迁移率的影响 (a) 窒温; (b) $77 \mathrm{~K}$

Fig. 6 Normalized 2DEG density $\left(\mathrm{N}_{\mathrm{S}}\right)$ and electron mobility $(\mu)$ versus channel thickness measured at (a) room-temperature and (b) 77K irradiated by electron beam

\section{4 讨 论}

InP HEMT外延材料的二维电子气来源于 InGaAs/InAlAs 量子阱, 其输运特性 主要受InGaAs/InAlAs 异质结构能带结构和远程电离杂质散射、库伦散射、合金 散射等各种散射过程的影响。二维电子气受到的散射越强, 电子迁移率越低。当 用高能电子束辐照InP HEMT外延材料时, 电子会和材料中的晶格原子相互作用, 发生弹性碰撞或非弹性碰撞, 将部分能量传给晶格原子, 导致晶格原子获得足够 能量, 克服周围原子束缚, 挤入晶格间隙, 同时原来的晶格位置成为空位, 形成 辐射诱导点缺陷 ${ }^{[23]}$. 且在注量不太高的情况下, 辐射诱导点缺陷浓度与电子注 量的大小成正比. 另外高能电子辐照也会导致沟道InGaAs/InAlAs 异质结附近区 域合金无序和远程离化杂质等辐射诱导缺陷增多。这些在 InP HEMT沟道

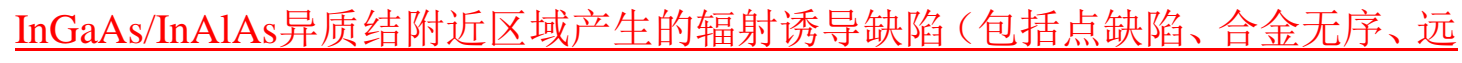
程离化杂质等缺陷) 和材料中的其他杂质会破坏晶格周期性, 在量子阱区域晶格 
禁带中产生局部分立缺陷能级，使载流子散射增强，导致InP HEMT外延材料二 维电子气（2DEG）电子迁移率减小, 其中部分缺陷能级成为载流子俘获中心, 俘获载流子后出现附加电荷, 削弱沟道InGaAs/InAlAs 量子阱异质界面内建电场, 降低能带弯曲程度, 使异质结界面处三角势变浅, 导致二维电子气浓度减小 ${ }^{[23]}$.

从图3和图4可以观察到 $\delta$ 掺杂浓度较高和隔离层较薄的InP HEMT外延材料 二维电子气浓度和电子迁移率辐照损伤相对较小。这主要是由于InP HEMT外延 结构材料二维电子气浓度随着 $\delta$ 掺杂浓度增加和隔离层厚度减少而迅速提高, 同 时二维电子气电子迁移率略微降低, 如表 2 中 A 组和B组数据所示。闹时在辐照剂 量相等且不是很高的条件下, 在材料中引入的辐照诱导缺陷数量基本相等, 导致 辐射诱导缺陷引起的二维电子浓度减小数目基本相同, 因此辐照后会导致较高 $\delta$ 掺杂浓度和较薄隔离层厚度的InP HEMT二维电子气浓度损伤相对较低. 此外, 低 $\delta$ 掺杂浓度和较厚隔离层的InP HEMT外延结构材料中困于引入的远程电离杂 质 $\mathrm{Si}$ 较少, 远程杂质散射较弱, 导致低 $\delta$ 掺杂浓度和较厚隔离层的InP HEMT二维 电子气迁移率较高, 如表 2 中A组和B组数据所示。由于辐照后会在InP HEMT沟 道InGaAs/InAlAs 异质结附近区域产生辐射诱导缺陷, 此时二维电子气的电子迁 移率主要由辐射诱导缺陷散射决定, 其它非辐照诱导散射如远程电离杂质散射、 合金散射、晶格散射等可以忽略不计。在相同辐照条件下，样品中引入的辐照诱 导缺陷数量基本相同, 从而引入的辐射诱导缺陷散射大致相同, 但是辐射诱导缺 陷散射会造成较低 $\delta$ 掺杂逨度和较厚隔离层厚度的InP HEMT二维电子气电子迁 移率减小的更多, 圈此辐照后会导致较高 $\delta$ 掺杂浓度和较薄隔离层厚度的 InP HEMT二维电和气迎移率损伤相对较低.

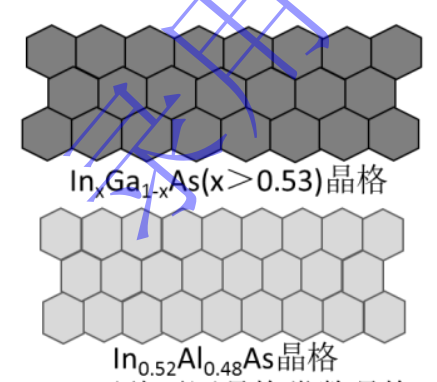

(a) 两种不同晶格常数晶格

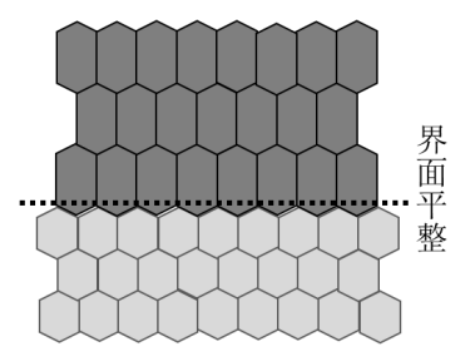

(b) 应变结

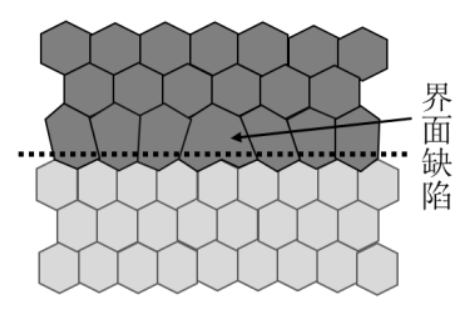

(c) 弛豫结

图7 InGaAs/InAlAs应变异质结的外延生长及弛豫示意图

Fig. 7 schematic diagram of epitaxial growth and relaxation of InGaAs/InAlAs strained heterojunction

从图 5 可以观察到沟道In组分较低的InP HEMT外延材料二维电子气辐照损 
伤相对较小。原因如下所述, 在晶格失配的沟道 $\mathrm{In}_{\mathrm{x}} \mathrm{Ga}_{1-\mathrm{x}} \mathrm{As} / \mathrm{InAlAs}$ 量子阱中, 晶 格的对称性由于应变层的引入发生变化, 外延层横向发生压缩应变使晶格常数与 祄底匹配, 即晶格常数在平行于异质结平面的两个方向相对原来缩小, 受到双轴 压应力, 同时纵向伸长发生张应变, 如图7(a)和7(b) InGaAs/InAlAs应变异质结的 外延生长示意图所示。在双轴压应变层中, 净的带隙移动为 ${ }^{[24]}$ :

$$
\Delta \mathrm{E}=-\alpha \cdot \varepsilon \geq 0,
$$

$\alpha$ 为形变势大于 0 , 与材料的弹性常数等有关; $\varepsilon$ 是压应变小于等于 0 . 从上面公 式可以看出随着压应变增大异质结净的带隙也会随着增大, 压应变如果消失, 净 的带隙移动也会跟着消失. 随着In组分的增大, 压应变增大, 沟道异质结界面势 阱变深, 量子阱二维电子气受到的散射减小, 电子迁移率恞速增夫, 同时电离施 主杂质电子也更容易转移到量子阱中, 导致二维电子气浓度也略微增大, 如表2 中C组样品数据所示. 在经过同等条件辐照后, 通过辐照诱导缺陷的引入, 部分 失配应变弛豫, 导致沟道 $\mathrm{n}_{\mathrm{x}} \mathrm{Ga} 1_{-\mathrm{x}} \mathrm{As}$ 应变层的晶格常数与祄底 $I n P$ 晶格常数不再相 等, 应变能量将通过在异质结界面附近产㜔错缺陷而释放出来, 如图7(c)所示. 因此辐射诱导缺陷会造成沟道In组分高的InP HEMT外延结构高应变量子阱晶格 严重弛豫, 导致量子阱势垒降低, 应变量子阱弛豫引起的位错散射和远程离化杂 质散射将会增强, 导致沟道In组分高的InP HEMT外延结构材料二维电子气迁移 率辐射损伤更大, 所以辐照后会导致沟道In组分低应变较小的InP HEMT二维电 子气电子迁移率损伤相对较低, 同时, 沟道异质结界面辐射诱导缺陷俘获载流子 后会出现附加电荷, 消弱导质界面内建电场, 使界面能带弯曲程度降低, 三角势 阱变浅, 造成维电子的载流子浓度降低 ${ }^{[23]}$. 同时应变驰豫后会导致量子阱 势垒进一步降低, 造成二维电子气的载流子浓度再次降低. 因此, 随着In组分增 大虽然右以略微增大二维电子气浓度, 但是随着辐照后应力弛豫同样会导致二维 电子气浓度损伤更大. 所以沟道In组分较低对InP HEMT二维电子气的辐射损伤 变化较小, 具有更好的抗辐照能力.

从图6可以观察到沟道厚度较厚的InP HEMT外延材料二维电子气辐照损伤 变化相对较小. 由于InP HEMT外延结构材料二维电子气浓度和电子迁移率随沟 道厚度变化不明显, 如表 2 所示. 在相同辐照剂量条件下, 在外延结构材料中引入 的辐照损伤缺陷数量基本一致, 导致辐射诱导缺陷复合的二维电子浓度减小数目 
基本相同, 沟道厚度对InP HEMT二维电子气浓度辐射损伤影响较小, 可以忽略 不计. 但是, 辐照在量子阱材料异质界面引入的辐照损伤较大, 造成辐射诱导缺 陷集聚在异质界面, 量子阱沟道厚度越小, 辐照后量子阱内的缺陷浓度越高, 量 子阱内的二维电子气受到的缺陷散射越强, 导致沟道厚度窄的InP HEMT二维电 子气迁移率损伤相对较大. 因此辐照后会导致沟道厚度较厚的InP HEMT二维电 子气电子迁移率损伤相对变化较小.

\section{5 结 论}

本文利用电子加速器试验装置, 开展了不同外延结构参数的 InP HEMT 外延 结构材料的电子辐射效应试验研究. 通过研究 InP HEMT 外延结构材料二维电 子气辐射损伤与外延结构参数 (列如 $\mathrm{Si}-\delta$ 掺杂浓度、InGaAs 沟道厚度和沟道 In 组分以及隔离层厚度等结构参数) 的依赖关系, 获得了不同外延结构 InP HEMT 材料二维电子气归一化浓度和电子迁移率随外延参数的变化规律. 值得注意的 是, 研究发现 $\delta$ 掺杂浓度越大, 隔离层厚度较溥, InGaAs 沟道厚度较大, 沟道 In 组分较低应变较小的 InP HEMT 外延结构䄰料二维电子气辐射损伤相对较低, 具有更强的抗辐照能力. 经分析认为原因如 传递, 产生弹性或非弹性碰撞, 且在 InR HEMT 沟道 InGaAs/InAlAs 异质结附近 区域产生辐射诱导缺陷, 破坏晶格完整性, 在量子阱区域晶格禁带中产生局部分 立缺陷能级, 增大复合中必密度, 散射增强导致二维电子气迁移率降低, 同时, 沟道异质结界面辐射诱导缺陷俘获载流子后会出现附加电荷, 削弱异质界面内建 电场, 使界面能带弯曲程度降低, 三角势阱变浅, 造成二维电子气的载流子浓度 降低. 2) 高浓度 Si $\delta$ 掺杂和薄隔离层有利于提高量子阱二维电子气浓度, 降低二 维电子气浓度受辐射损伤的影响. 3) 高 In 组分应变沟道有利于提高二维电子气 迁移率, 但辐照后更容易应变驰豫释放应变能量在异质结界面产生位错缺陷, 导 致二维电子气迁移率显著下降, 此外应变弛豫还会同时导致量子阱势垒降低, 导 致二维电子浓度进一步降低.

\section{参考文献:}

[1] Cha E, Wadefalk N, Moschetti G, Pourkabirian A, Stenarson J, Grahn J 2020 IEEE Electron Device Lett. 411005

[2] Hamada H, Tsutsumi T, Matsuzaki H, Fujimura T, Abdo I, Shirane A, Okada K, Itami G, Song H J, Sugiyama H, Nosaka H 2020 IEEE J. Solid-State Circuits 552316 
[3] Sato M, Niida Y, Suzuki T, Nakasha Y, Kawano Y, Iwai T, Hara N, Joshin K 2017 IEICE Trans. Electron. E100C 417

[4] Tang J J, Liu G P, Zhao G J, Xing S, Malik S A 2020 J. Vac. Sci. Technol. B 38023202

[5] Tang J J, Liu G P, Song J Y, Zhao G J, Yang J H 2021 Chin. Phys. B 30027303

[6] Gu W, Zhang J, Wang C, Feng Q, Ma X-H, Hao Y 2009 Acta Phys. Sin. 581161

[7] Ives N E, Chen J, Witulski A F, Schrimpf R D, Fleetwood D M, Bruce R W, McCurdy M W, Zhang E X, Massengill L W 2015 IEEE Trans. Nucl. Sci. 622417

[8] Fleetwood D M 2015 IEEE Trans. Nucl. Sci. 621462

[9] Sun S X, Ding P, Jin Z, Zhong Y H, Li Y X, Wei Z C 2019 Nanomaterials 9967

[10] Daoudi M, Kaouach H, Hosni F 2016 Optik 1277188

[11] Chen R, Liang Y, Han J, Wang X, Yang H, Chen Q, Yuan R, Ma Y, Shangguan S 2021 Acta Phys. Sin. 70116102 (in Chinese) [陈睿, 梁亚楠, 韩建伟, 王璇, 杨涵, 陈钱, 袁润杰, 马 英起，上官士鹏 2021 物理学报 70 116102]

[12] Sun S X, Yang B, Zhong Y H, Li Y X, Ding P, Jin Z, Wei Z C 2020 J/Rhys! D: Appl. Phys. 53 175107

[13] Warner J H, McMorrow D, Buchner S, Boos J B, Roche N, Páillet P, Gaillardin M, Blackmore E, Trinczek M, Ramachandran V, Reed R A, Schrimpf R D2013 IEEE Trans. Nucl. Sci. 602651

[14] Pearton S J, Ren F, Patrick E, Law M E, Polyakov A Y2016 ECS J. Solid State Sci. Technol. 5 Q35

[15] Lin L, Liao Z, Liu Q, Lu T, Feng X 2002 Surf, Coat. Technol. 158737

[16] Lin L, Li Y, Chen W, Jiang J 1995 Journal of Sichuan University(Natural Science Edition) 32 39 (in Chinese) [林理涁, 李有梅, 陈卫东, 蒋锦红 1995 四川大学学报 (自然科学版) 3239

[17] Jinjin T, Guipeng L, Bangyao M, Salamat A, Guijuan Z, Jianhong Y 2021 Phys. Lett. A 410 127527

[18] Zhang Z, Cardwell D, Sasikumar A, Kyle E C H, Chen J, Zhang E X, Fleetwood D M, Schrimpf R D, Speck J S, Arehart A R, Ringel S A 2016 J. Appl. Phys. 119165704

[19] Smith M D, O'Mahony D, Vitobello F, Muschitiello M, Costantino A, Barnes A R, Parbrook P J 2016 Semicond. Sci. Technol. 31025008

[20] Zhong Y H, Yang B, Chang M M, Ding P, Ma L H, Li M K, Duan Z Y, Yang J, Jin Z, Wei Z C 2020 Chin. Phys. B 29038502

[21] Shu-Xing Z, Ming Q, Li-Kun A, An-Huai X, Li-Dan W, Peng D, Zhi J 2015 Chin. Phys. Lett. 32116

[22] Klaassen D B M 1992 Solid-State Electron. 35961

[23] Ma D, Li Y, Guo Q, Ai E, Wang H, Zeng J 2015 Acta Phys. Sin. 64154217 (in Chinese) [玛丽 䍀，柰豫东，郭旗，艾尔肯，王海娇，汪波，曾骏哲 2015 物理学报 64 154217]

[24] Anderson N G, Laidig W D, Kolbas R M, Lo Y C 1986 J. Appl. Phys. 602361 


\section{Structure parameters design of InP HEMT epitaxial materials to}

\section{improve the radiation-hardened ability*}

Zhou Shu-Xing ${ }^{1)^{\dagger}}$ Fang Ren-Feng ${ }^{1)}$ Wei Yan-Feng ${ }^{1)}$ Chen Chuan-Liang ${ }^{1)}$ Cao Wen-Yu ${ }^{1)}$ Zhang $\mathrm{Xin}^{1)} \mathrm{Ai}$ Li-Kun ${ }^{2)} \mathrm{Li}$ Yu-Dong ${ }^{3)}$ Guo Qi ${ }^{3)}$

1) (Hubei Key Laboratory of Low Dimensional Optoelectronic Materials and Devices, Hubei University of Arts and Science, Xiangyang 441053, China)

2) (State Key Laboratory of Functional Materials for Informatics, Shanghai Institute of Microsystem and Information Technology, Chinese Academy of Sciences, Shanghai 200050,

China)

3) (Xinjiang Technical Institute of Physics and Chemistry, Chinese Academy of Sciences, Urumqi 830011, China)

\section{Abstract}

In order to improve the radiation-hardened ability of the InP HEMT by optimizing the epitaxial structure design, a series of InP HEMT epitaxial structure materials with different structure parameters were grown by gas source molecular beam epitaxy (GSMBE). These samples are irradiated at room temperature by $1.5 \mathrm{MeV}$ electron beam at the same irradiation fluence of $2 \times 10^{15} \mathrm{~cm}^{2}$. The electrical properties of the twodimensional electron gas (2DEG) for InP HEMT epitaxial materials before and after irradiation are measured by hall measurements to obtain the changes of the normalized 2DEG density and electron mobility along with the epitaxial structure parameters. The relationship between 2DEG radiation damage and epitaxial structure parameters (such as $\mathrm{Si}$ - $\delta$-doping density spacer thickness, channel thickness and channel In content) of InP HEMT epitaxial structure materials was analyzed. The results show that the 2DEG of the InP HEMT epitaxial structure material with higher Si- $\delta$-doping density, thinner spacer thickness, thicker channel thickness and lower channel In content has lower radiation damage, which earned a stronger radiation-hardened ability.

Keywords: InP HEMT; two-dimensional electron gas; electron beam irradiation; radiation hardening

PACS: 72.80.Ey, 71.10.Ca, 61.82.-d, 61.80.-x

* Project supported by the National Natural Science Foundation of China (Grant No. 11705277, 61434006) and the Doctoral Research Foundation Project of Hubei University of Arts and Science (kyqdf2059038).

†Corresponding author. E-mail: sxzhou2020@163.com 